\title{
Chemotherapy Response Score
}

National Cancer Institute

\section{Source}

National Cancer Institute. Chemotherapy Response Score. NCI Thesaurus. Code C159367.

A three-tier chemotherapy response score (CRS) system used to assess the histopathologic response and prognosis of patients with tubo-ovarian high-grade serous carcinoma (HGSC) receiving neoadjuvant chemotherapy. 